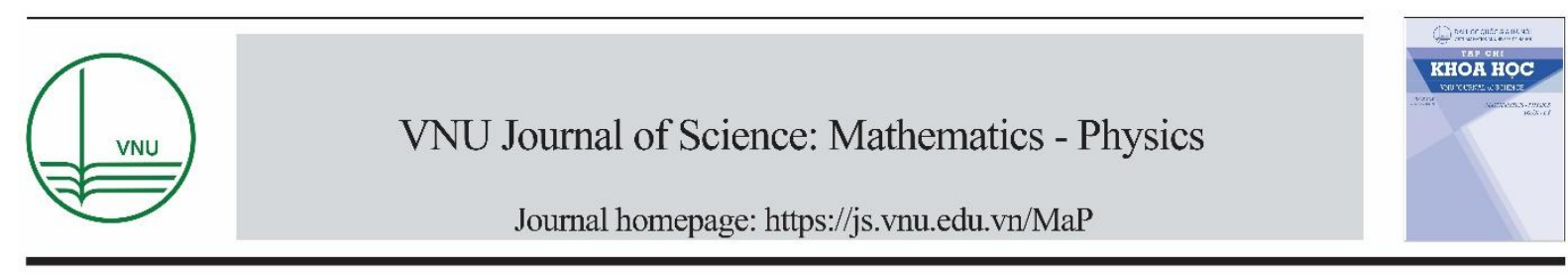

Original Article

\title{
Neutron Cross Section Generation for PWR MOX Fuel Assemblies with SCALE and Serpent
}

\author{
Vu Thanh Mai ${ }^{1}$, Donny Hartanto ${ }^{2}$, Tran The Anh ${ }^{1}$, \\ Luu Thi Lan ${ }^{3}$, Tran Viet $\mathrm{Phu}^{4}$, Pham Nhu Viet $\mathrm{Ha}^{4, *}$ \\ ${ }^{I}$ VNU University of Science, 334 Nguyen Trai, Thanh Xuan, Hanoi, Vietnam \\ ${ }^{2}$ University of Sharjah, P.O.BOX, 27272, Sharjah, United Arab Emirates \\ ${ }^{3}$ Vietnam National Cancer Hospital, 30 Cau Buou, Thanh Tri, Hanoi, Vietnam \\ ${ }^{4}$ Institute for Nuclear Science and Technology, VINATOM, 179 Hoang Quoc Viet, Hanoi, Vietnam
}

Received 07 August 2020

Revised 28 August 2020; Accepted 09 September 2020

\begin{abstract}
In this study, the SCALE/TRITON code (based on deterministic method) and the Serpent 2 code (based on Monte Carlo method) were utilized to prepare the group constants of the pressurized water reactor (PWR) mixed-oxide (MOX) fuel assemblies for transient analyses of PWR MOX fueled cores in normal operation and control rod ejection accident condition with 3D reactor kinetics codes. The PWR MOX fuel assemblies were modeled with TRITON and Serpent, and their infinite neutron multiplication factors ( $\mathrm{k}$-inf) versus burnup and respective two-group neutron cross sections were calculated and compared against the available benchmark data obtained with the HELIOS code. The comparative results generally show a good agreement between TRITON and Serpent with HELIOS within $643 \mathrm{pcm}$ for the k-inf values and within 5\% for the two-group neutron cross sections. Therefore, it indicates that the TRITON and Serpent models developed herein for the PWR MOX fuel assemblies can be applied to group constant generation to be further used in transient analyses of PWR MOX fueled cores.
\end{abstract}

Keywords: PWR MOX fuel assembly, group constant, SCALE, Serpent.

\section{Introduction}

The utilization of recycled plutonium as mixed-oxide (MOX) nuclear fuel in light water reactor (LWR) cores helps to save the natural uranium resources and reduce either the amount of weapon-grade

\footnotetext{
* Corresponding author.

Email address: phamha@ vinatom.gov.vn
}

https//doi.org/ 10.25073/2588-1124/vnumap.4596 
plutonium or the plutonium amount, which has to be disposed to the final storage. However, the control rod ejection accident (REA), which is a consequence of mechanical failure of the control rod drive mechanism casing located on the reactor pressure vessel top and categorized as design-basis reactivityinitiated accident in pressurized water reactors (PWRs), is of particular concern for MOX fueled cores since their delayed neutron fractions are significantly smaller than those of $\mathrm{UO}_{2}$ cores [1]. The rod ejection transient can result in localized perturbations of the neutronic and thermal-hydraulic core parameters, which can be difficult for reactor core simulators to predict precisely, especially in a heterogeneous $\mathrm{MOX} / \mathrm{UO}_{2}$ fueled core. In this regard, the OECD/NEA and U.S. NRC PWR MOX/UO Core Transient Benchmark [1] has been well defined with a complete set of PWR MOX/UO $\mathrm{UO}_{2}$ core steady-state and transient benchmark input and unique solutions. The purpose of the benchmark was to provide the framework to assess the ability of the heterogeneous transport and nodal diffusion transient methods and codes like the 3D reactor kinetic code PARCS [2] to predict the control rod ejection transient response of a core partially loaded with weapons grade MOX fuel. It is noted that the core chosen for the simulation is based on a four-loop Westinghouse PWR similar to the reactor chosen for plutonium disposition in the U.S.

In this research, we aim to use the 3D reactor kinetics codes like PARCS and NODAL3 [3] to examine thoroughly REAs in the PWR MOX fueled core taken from the OECD/NEA and U.S. NRC PWR MOX/UO ${ }_{2}$ Core Transient Benchmark [1]. Therefore, the present study is performed to generate the two-group neutron cross sections for the PWR MOX fuel assemblies using both the deterministic and Monte Carlo methods to serve for further steady-state and transient calculations of the PWR MOX core with PARCS and NODAL3. The TRITON module (based on deterministic method) of the SCALE code system [4] and the Serpent 2 code (based on Monte Carlo method) [5] were utilized herein to do this homogenized two-group neutron cross section generation. The infinite neutron multiplication factors of the PWR MOX fuel assemblies versus burnup and the respective neutron cross sections obtained with TRITON and Serpent were analyzed in relation to the available benchmark data.

\section{Calculation Models and Methods}

The PWR MOX fuel assembly configuration was obtained from Ref. [1] with 17x17 fuel lattice including 264 fuel rods, 24 Wet Annular Burnable Absorber (WABA) pins, and a guide tube as shown in Figure 1. The heavy metal composition in the PWR MOX 4.0\% and MOX 4.3\% fuel assemblies is represented in Table 1 . The detailed fuel assembly design parameters, material composition, feedback data and related benchmark data can be found in Ref. [1]. In the present work, the PWR MOX 4.0\% and MOX $4.3 \%$ fuel assembly types were modelled and analysed with the SCALE/TRITON module (deterministic method) and Serpent 2 (Monte Carlo method). It is recalled that the deterministic and Monte lattice physics codes SCALE/TRITON [4] and Serpent 2 [5] have been widely used to generate homogenized multi-group neutron cross-sections for full-core calculations of large power reactors like the PWRs. These multi-group constants are generally required for multi-group nodal diffusion transient codes like PARCS and NODAL3 to predict the behaviour of a reactor core under steady-state and transient conditions.

To generate multi-group neutron cross sections, the TRITON module called XSDRNPM, which is a discrete-ordinates code, is applied to solve the one-dimensional Boltzmann neutron transport equation in slab, cylindrical, or spherical coordinates [4]. XSDRNPM will use the fluxes determined from its spectral calculation to collapse input cross sections and write these into one of several formats. Meanwhile, the Monte Carlo code Serpent 2 produces the homogenized few-group cross sections by the two steps. First, multi-group neutron cross sections are calculated by using the Monte Carlo tallies. Second, the few-group neutron cross sections are generated by condensing the previously calculated 
multi-group cross sections using different options such as infinite spectrum and B1 leakage corrected spectrum [6]. Besides, additional methods have been adopted in Serpent 2 to produce diffusion coefficients or transport cross sections such as the cumulative migration method [7] and by applying user-defined transport correction factors [8], e.g., for reactor cores with substantial neutron leakage. In this investigation, the B1 leakage corrected spectrum approach was applied with SCALE/TRITON and Serpent to obtain the few-group transport cross sections of the PWR MOX fuel assemblies.

The PWR MOX fuel assembly models with SCALE/TRITON and Serpent are described in Figures 2 and 3, respectively. The two models have the same geometry in two dimensions with infinite assembly height and the same material composition. The only differences are the transport and depletion solution methods as well as the nuclear data libraries used in each code. The SCALE 252-group library and the continuous-energy ENDF/B-VII.0 library [9] were used in the TRITON and Serpent calculations, respectively. In the calculation with Serpent, the WIMS 172 energy group structure [10] was used as the intermediate multi-group structure for the generation of the two-group neutron cross-sections. It is noted that the main TRITON and Serpent calculations were done under the hot full power condition (fuel temperature is $900 \mathrm{~K}$, moderator temperature is $580 \mathrm{~K}$ and boron concentration is $1000 \mathrm{ppm}$ ). Additionally, the two-group neutron cross sections of the PWR MOX 4.0\% and MOX $4.3 \%$ fuel assemblies also need to be generated by TRITON and Serpent for various branch conditions encompassing different fuel temperatures $(560 \mathrm{~K}, 900 \mathrm{~K}$ and $1320 \mathrm{~K})$, different moderator temperatures $(560 \mathrm{~K}, 580 \mathrm{~K}$ and $600 \mathrm{~K})$, different boron concentrations $(0 \mathrm{ppm}, 1000 \mathrm{ppm}$ and $2000 \mathrm{ppm})$ and different burnup levels $(0.15 \mathrm{GWd} / \mathrm{tHM}$ and $37.5 \mathrm{GWd} / \mathrm{tHM})$ to cover the expected range of PWR MOX fuelled core operating conditions. The branch calculation mode was therefore utilized in the TRITON and Serpent calculations in order to obtain these two-group neutron cross sections. Finally, the infinite neutron multiplication factors (k-inf) of the PWR MOX fuel assemblies versus burnup and the respective two-group neutron cross sections obtained with TRITON and Serpent were analyzed in relation to the reference benchmark data obtained by the deterministic code HELIOS with a 47-group library as presented and discussed in the following section.

Table 1. Heavy metal (HM) composition in the PWR MOX fuel assemblies.

\begin{tabular}{|c|c|c|c|}
\hline Assembly type & Density $\left[\mathrm{g} / \mathrm{cm}^{3}\right]$ & \multicolumn{2}{|c|}{ HM material } \\
\hline \multirow[t]{3}{*}{ MOX $4.0 \%$} & 10.41 & $\begin{array}{l}\text { Corner zone: } \\
2.5 \mathrm{wt} \% \mathrm{Pu} \text {-fissile }\end{array}$ & \multirow{3}{*}{$\begin{array}{c}\text { Uranium vector: } \\
234 / 235 / 236 / 238= \\
0.002 / 0.2 / 0.001 / 99.797 \mathrm{wt} \%\end{array}$} \\
\hline & & $\begin{array}{l}\text { Peripheral zone: } \\
3.0 \mathrm{wt} \% \text { Pu-fissile }\end{array}$ & \\
\hline & & $\begin{array}{l}\text { Central zone: } \\
4.5 \mathrm{wt} \% \text { Pu-fissile }\end{array}$ & \\
\hline \multirow[t]{3}{*}{ MOX $4.3 \%$} & 10.41 & $\begin{array}{l}\text { Corner zone: } \\
2.5 \mathrm{wt} \% \text { Pu-fissile }\end{array}$ & \multirow{3}{*}{$\begin{array}{c}\text { Plutonium vector: } \\
\text { 239/240/241/242= } \\
93.6 / 5.9 / 0.4 / 0.1 \mathrm{wt} \%\end{array}$} \\
\hline & & $\begin{array}{l}\text { Peripheral zone: } \\
3.0 \mathrm{wt} \% \mathrm{Pu} \text {-fissile }\end{array}$ & \\
\hline & & $\begin{array}{l}\text { Central zone: } \\
5.0 \mathrm{wt} \% \text { Pu-fissile }\end{array}$ & \\
\hline
\end{tabular}




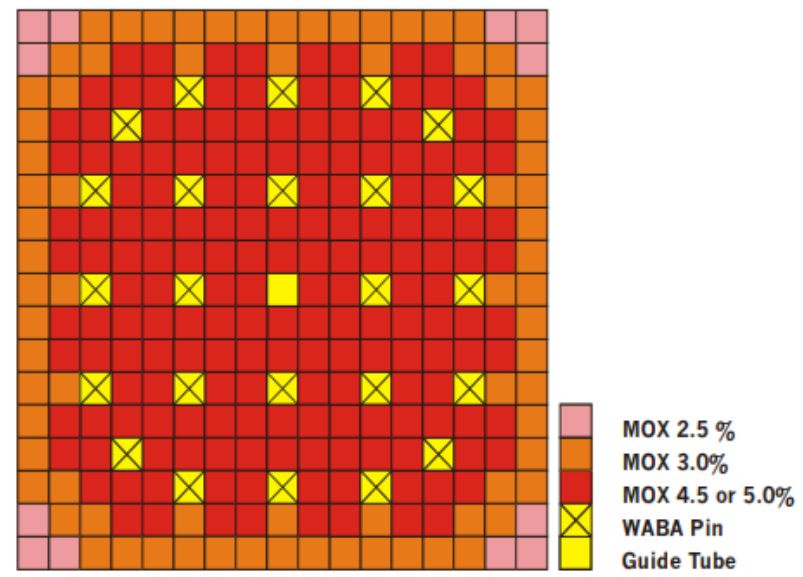

Figure 1. PWR MOX fuel assembly configuration [1].

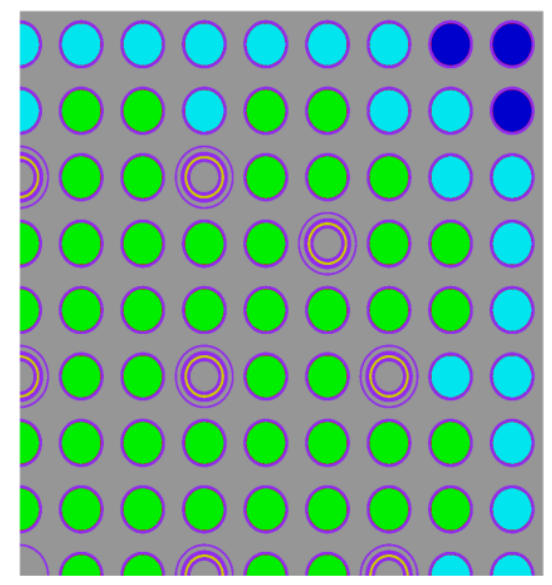

Figure 2. PWR MOX fuel assembly model (one-fourth symmetry) with SCALE/TRITON.

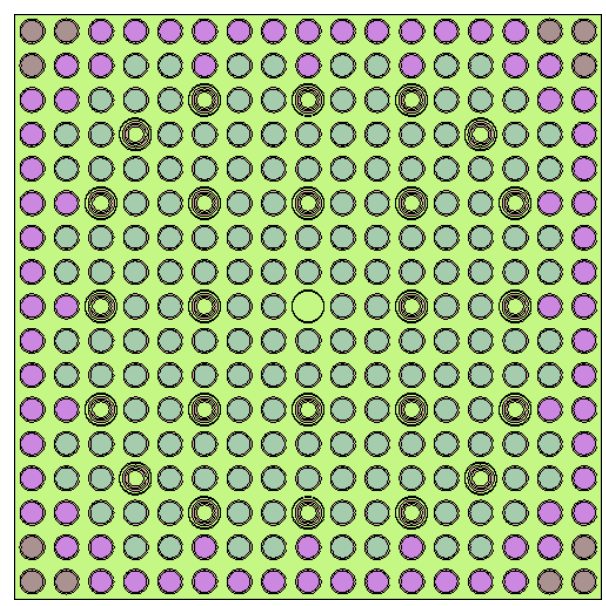

Figure 3. PWR MOX fuel assembly model with Serpent. 


\section{Results and Discussion}

The k-inf values of the PWR MOX 4.0\% and MOX $4.3 \%$ fuel assemblies as a function of fuel burnup obtained with TRITON and Serpent are presented in Figures 4 and 5 in comparison with the reference benchmark data obtained with the deterministic code HELIOS [1]. It can be seen that there is generally a good agreement between the three codes in predicting the k-inf value of the PWR MOX assemblies versus burnup. For the PWR MOX $4.0 \%$ fuel assembly, the maximum deviations of the TRITON and Serpent results from the benchmark data were $479 \mathrm{pcm}$ and $564 \mathrm{pcm}$, respectively. Meanwhile, these maximum values were $588 \mathrm{pcm}$ and $643 \mathrm{pcm}$ for the PWR MOX $4.3 \%$ fuel assembly. As the content of the plutonium in the central zone of the MOX assembly slightly increased from 4.5 $\mathrm{wt} \%$ for the MOX $4.0 \%$ assembly to $5.0 \mathrm{wt} \%$ for the MOX $4.5 \%$ assembly (see Table 1), it was observed from Figures 4 and 5 that the disagreement of the three codes in predicting the k-inf value of the MOX assembly versus burnup slightly increased. In addition, as the fuel burns out, the TRITON results tend to overestimate the benchmark values while the Serpent results tend to underestimate the benchmark data. Moreover, it is noticed that the k-inf values of the MOX assemblies predicted using Monte Carlo method with Serpent underestimated those predicted using deterministic methods with TRITON and HELIOS.

The homogenized two-group neutron cross sections of the PWR MOX $4.0 \%$ and MOX $4.3 \%$ fuel assemblies were also generated using TRITON and Serpent for various reactor operating conditions, i.e., branch conditions, at different fuel temperatures $(560 \mathrm{~K}, 900 \mathrm{~K}$ and $1320 \mathrm{~K})$, different moderator temperatures $(560 \mathrm{~K}, 580 \mathrm{~K}$ and $600 \mathrm{~K})$, different boron concentrations $(0 \mathrm{ppm}, 1000 \mathrm{ppm}$ and $2000 \mathrm{ppm})$ and different burnup levels $(0.15 \mathrm{GWd} / \mathrm{tHM}$ and $37.5 \mathrm{GWd} / \mathrm{tHM})$. These two-group neutron cross sections obtained with TRITON and Serpent were compared with the benchmark data generated by the deterministic code HELIOS [1]. For the MOX 4.0\% assembly, the comparison is illustrated in Table 2 for the main two-group constants including the transport, absorption and fission production (nu*fission) cross sections at different fuel temperatures, moderator temperatures and boron concentrations at the burnup of $0.15 \mathrm{GWd} / \mathrm{tHM}$ and in Table 3 for those cross sections at the burnup of $37.5 \mathrm{GWd} / \mathrm{tHM}$. Similarly, these cross sections for the MOX $4.3 \%$ assembly at the burnup of $0.15 \mathrm{GWd} / \mathrm{tHM}$ are represented in Table 4 and those at the burnup of $37.5 \mathrm{GWd} / \mathrm{tHM}$ are listed in Table 5.

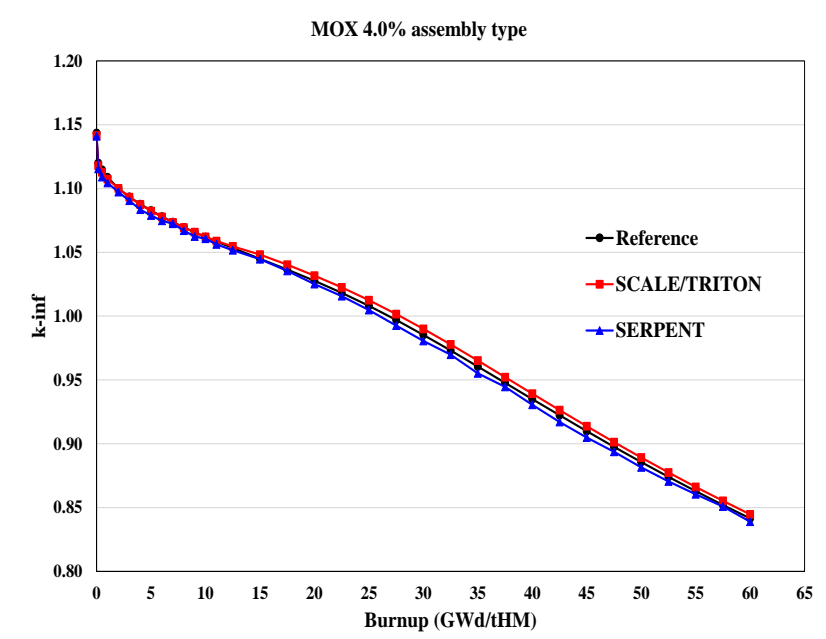

Figure 4. The k-inf of the PWR MOX $4.0 \%$ fuel assembly versus burnup. 


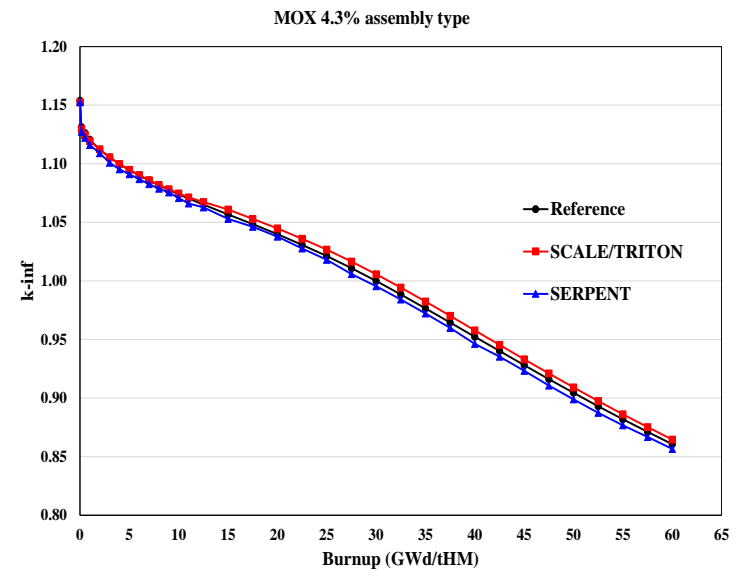

Figure 5. The k-inf of the PWR MOX 4.3\% fuel assembly versus burnup.

It was found that the homogenized two-group neutron cross sections of the PWR MOX $4.0 \%$ and 4.3\% fuel assemblies generated by TRITON and Serpent generally compared well within 5\% with the benchmark data obtained with HELIOS as illustrated in Tables 2-5. However, the deviations of the twogroup transport cross sections predicted by TRITON and Serpent from the benchmark values were within $6-16 \%$ for the MOX $4.0 \%$ assembly and within $7-19 \%$ for the MOX $4.3 \%$ assembly. Since the same geometry and material composition of the PWR MOX fuel assemblies as given in the benchmark [1] were used in the calculations, the discrepancy between the three codes, especially in predicting the two-group transport cross sections, might be largely due to different transport and depletion solution methods and nuclear data libraries employed in each code. It is also noted that the two-group neutron cross sections obtained with TRITON and Serpent generally agreed well within few percents with each other. Furthermore, different methods for precise generation of the diffusion coefficients or the transport cross sections of the PWR MOX assemblies, e.g., the B1 leakage corrected spectrum approach (used in this study) and the cumulative migration method [7], are being considered in future work to identify the most suitable one for control rod ejection transient analyses of the PWR MOX fueled cores with 3D reactor kinetics codes.

Table 2. Two-group cross sections of the PWR MOX $4.0 \%$ fuel assembly at $0.15 \mathrm{GWd} / \mathrm{tHM}$.

\begin{tabular}{|l|c|c|c|c|}
\hline \multicolumn{5}{|c|}{ Fuel temperature $\mathbf{9 0 0 K}$; moderator temperature $\mathbf{5 8 0 K}$; boron concentration $\mathbf{~ 1 0 0 0} \mathbf{~ p p m}$} \\
\hline & Group & Transport & Absorption & Nu*Fission \\
\hline Reference & 1 & $2.35776 \mathrm{E}-01$ & $1.17228 \mathrm{E}-02$ & $8.30529 \mathrm{E}-03$ \\
\hline SCALE/TRITON & 1 & $2.14169 \mathrm{E}-01$ & $1.20205 \mathrm{E}-02$ & $8.39047 \mathrm{E}-03$ \\
\hline SERPENT & 1 & $2.14223 \mathrm{E}-01$ & $1.19110 \mathrm{E}-02$ & $8.33229 \mathrm{E}-03$ \\
\hline Reference & 2 & $8.65492 \mathrm{E}-01$ & $2.50779 \mathrm{E}-01$ & $3.68848 \mathrm{E}-01$ \\
\hline SCALE/TRITON & 2 & $1.00024 \mathrm{E}+00$ & $2.39600 \mathrm{E}-01$ & $3.60164 \mathrm{E}-01$ \\
\hline SERPENT & 2 & $9.98201 \mathrm{E}-01$ & $2.49794 \mathrm{E}-01$ & $3.66092 \mathrm{E}-01$ \\
\hline \multicolumn{2}{|c|}{ Fuel temperature } & $\mathbf{9 0 0 K}$; moderator temperature $=\mathbf{5 8 0 K}$; boron concentration $=\mathbf{2 0 0 0} \mathbf{p p m}$ \\
\hline Reference & 1 & $2.35195 \mathrm{E}-01$ & $1.19489 \mathrm{E}-02$ & $8.31447 \mathrm{E}-03$ \\
\hline SCALE/TRITON & 1 & $2.14091 \mathrm{E}-01$ & $1.21884 \mathrm{E}-02$ & $8.37428 \mathrm{E}-03$ \\
\hline SERPENT & 1 & $2.14596 \mathrm{E}-01$ & $1.21479 \mathrm{E}-02$ & $8.34564 \mathrm{E}-03$ \\
\hline Reference & 2 & $8.63222 \mathrm{E}-01$ & $2.60169 \mathrm{E}-01$ & $3.68846 \mathrm{E}-01$ \\
\hline SCALE/TRITON & 2 & $1.00180 \mathrm{E}+00$ & $2.49164 \mathrm{E}-01$ & $3.60116 \mathrm{E}-01$ \\
\hline SERPENT & 2 & $9.94692 \mathrm{E}-01$ & $2.49328 \mathrm{E}-01$ & $3.65436 \mathrm{E}-01$ \\
\hline
\end{tabular}




\begin{tabular}{|l|c|c|c|c|}
\hline \multicolumn{5}{|c|}{ Fuel temperature $\mathbf{9 0 0 K}$; moderator temperature $=\mathbf{6 0 0 K}$; boron concentration $\mathbf{1 0 0 0} \mathbf{~ p p m}$} \\
\hline Reference & 1 & $2.27958 \mathrm{E}-01$ & $1.15443 \mathrm{E}-02$ & $8.22141 \mathrm{E}-03$ \\
\hline SCALE/TRITON & 1 & $2.08062 \mathrm{E}-01$ & $1.18480 \mathrm{E}-02$ & $8.30606 \mathrm{E}-03$ \\
\hline SERPENT & 1 & $2.08310 \mathrm{E}-01$ & $1.17459 \mathrm{E}-02$ & $8.24828 \mathrm{E}-03$ \\
\hline Reference & 2 & $8.14207 \mathrm{E}-01$ & $2.49066 \mathrm{E}-01$ & $3.68249 \mathrm{E}-01$ \\
\hline SCALE/TRITON & 2 & $9.45743 \mathrm{E}-01$ & $2.37814 \mathrm{E}-01$ & $3.59098 \mathrm{E}-01$ \\
\hline SERPENT & 2 & $9.42521 \mathrm{E}-01$ & $2.47859 \mathrm{E}-01$ & $3.65319 \mathrm{E}-01$ \\
\hline \multicolumn{4}{|c|}{ Fuel temperature $\mathbf{= 1 3 2 0 K} ;$ moderator temperature $=\mathbf{5 8 0 K}$; boron concentration $=\mathbf{1 0 0 0} \mathbf{p p m}$} \\
\hline Reference & 1 & $2.35581 \mathrm{E}-01$ & $1.19719 \mathrm{E}-02$ & $8.32281 \mathrm{E}-03$ \\
\hline SCALE/TRITON & 1 & $2.13977 \mathrm{E}-01$ & $1.24222 \mathrm{E}-02$ & $8.38588 \mathrm{E}-03$ \\
\hline SERPENT & 1 & $2.14273 \mathrm{E}-01$ & $1.21640 \mathrm{E}-02$ & $8.35630 \mathrm{E}-03$ \\
\hline Reference & 2 & $8.66167 \mathrm{E}-01$ & $2.51979 \mathrm{E}-01$ & $3.70746 \mathrm{E}-01$ \\
\hline SCALE/TRITON & 2 & $1.00300 \mathrm{E}+00$ & $2.50205 \mathrm{E}-01$ & $3.61734 \mathrm{E}-01$ \\
\hline SERPENT & 2 & $9.99312 \mathrm{E}-01$ & $2.50795 \mathrm{E}-01$ & $3.67749 \mathrm{E}-01$ \\
\hline
\end{tabular}

Table 3. Two-group cross sections of the PWR MOX $4.0 \%$ fuel assembly at $37.5 \mathrm{GWd} / \mathrm{tHM}$.

\begin{tabular}{|l|c|c|c|c|}
\hline \multicolumn{5}{|c|}{ Fuel temperature $=\mathbf{9 0 0 K}$; moderator temperature $=\mathbf{5 8 0 K}$; boron concentration $=\mathbf{1 0 0 0} \mathbf{~ p p m}$} \\
\hline & Group & Transport & Absorption & Nu*Fission \\
\hline Reference & 1 & $2.32911 \mathrm{E}-01$ & $1.26497 \mathrm{E}-02$ & $6.24237 \mathrm{E}-03$ \\
\hline SCALE/TRITON & 1 & $2.164380 \mathrm{E}-01$ & $1.26159 \mathrm{E}-02$ & $6.21028 \mathrm{E}-03$ \\
\hline SERPENT & 1 & $2.18325 \mathrm{E}-01$ & $1.28916 \mathrm{E}-02$ & $6.19141 \mathrm{E}-03$ \\
\hline Reference & 2 & $8.59974 \mathrm{E}-01$ & $1.64156 \mathrm{E}-01$ & $2.25377 \mathrm{E}-01$ \\
\hline SCALE/TRITON & 2 & $9.45816 \mathrm{E}-01$ & $1.56960800 \mathrm{e}-01$ & $2.24311 \mathrm{E}-01$ \\
\hline SERPENT & 2 & $9.42149 \mathrm{E}-01$ & $1.63357 \mathrm{E}-01$ & $2.22395 \mathrm{E}-01$ \\
\hline
\end{tabular}

Fuel temperature $=900 \mathrm{~K}$; moderator temperature $=580 \mathrm{~K}$; boron concentration $=2000 \mathrm{ppm}$

\begin{tabular}{|l|c|c|c|c|}
\hline Reference & 1 & $2.31966 \mathrm{E}-01$ & $1.28944 \mathrm{E}-02$ & $6.25413 \mathrm{E}-03$ \\
\hline SCALE/TRITON & 1 & $2.16360 \mathrm{E}-01$ & $1.27713 \mathrm{E}-02$ & $6.20071 \mathrm{E}-03$ \\
\hline SERPENT & 1 & $2.18575 \mathrm{E}-01$ & $1.31408 \mathrm{E}-02$ & $6.19881 \mathrm{E}-03$ \\
\hline Reference & 2 & $8.56066 \mathrm{E}-01$ & $1.73598 \mathrm{E}-01$ & $2.25445 \mathrm{E}-01$ \\
\hline SCALE/TRITON & 2 & $9.46170 \mathrm{E}-01$ & $1.66672 \mathrm{E}-01$ & $2.24447 \mathrm{E}-01$ \\
\hline SERPENT & 2 & $9.43434 \mathrm{E}-01$ & $1.73058 \mathrm{E}-01$ & $2.22779 \mathrm{E}-01$ \\
\hline
\end{tabular}

Fuel temperature $=900 \mathrm{~K}$; moderator temperature $=600 \mathrm{~K}$; boron concentration $=1000 \mathrm{ppm}$

\begin{tabular}{|l|l|l|l|l|}
\hline Reference & 1 & $2.25317 \mathrm{E}-01$ & $1.24405 \mathrm{E}-02$ & $6.17947 \mathrm{E}-03$ \\
\hline SCALE/TRITON & 1 & $2.10320 \mathrm{E}-01$ & $1.24096 \mathrm{E}-02$ & $6.14424 \mathrm{E}-03$ \\
\hline SERPENT & 1 & $2.12278 \mathrm{E}-01$ & $1.26892 \mathrm{E}-02$ & $6.11919 \mathrm{E}-03$ \\
\hline Reference & 2 & $8.07265 \mathrm{E}-01$ & $1.62782 \mathrm{E}-01$ & $2.25538 \mathrm{E}-01$ \\
\hline SCALE/TRITON & 2 & $8.92540 \mathrm{E}-01$ & $1.55654 \mathrm{E}-01$ & $2.24023 \mathrm{E}-01$ \\
\hline SERPENT & 2 & $8.86897 \mathrm{E}-01$ & $1.62099 \mathrm{E}-01$ & $2.22811 \mathrm{E}-01$ \\
\hline
\end{tabular}

Fuel temperature $=1320 \mathrm{~K}$; moderator temperature $=580 \mathrm{~K}$; boron concentration $=1000 \mathrm{ppm}$

\begin{tabular}{|l|c|c|c|c|}
\hline Reference & 1 & $2.32676 \mathrm{E}-01$ & $1.28949 \mathrm{E}-02$ & $6.23320 \mathrm{E}-03$ \\
\hline SCALE/TRITON & 1 & $2.16329 \mathrm{E}-01$ & $1.28368 \mathrm{E}-02$ & $6.19575 \mathrm{E}-03$ \\
\hline SERPENT & 1 & $2.18270 \mathrm{E}-01$ & $1.31249 \mathrm{E}-02$ & $6.17347 \mathrm{E}-03$ \\
\hline Reference & 2 & $8.60198 \mathrm{E}-01$ & $1.64779 \mathrm{E}-01$ & $2.26582 \mathrm{E}-01$ \\
\hline SCALE/TRITON & 2 & $9.46279 \mathrm{E}-01$ & $1.57559 \mathrm{E}-01$ & $2.25356 \mathrm{E}-01$ \\
\hline SERPENT & 2 & $9.42152 \mathrm{E}-01$ & $1.63947 \mathrm{E}-01$ & $2.23534 \mathrm{E}-01$ \\
\hline
\end{tabular}


Table 4. Two-group cross sections of the PWR MOX $4.3 \%$ fuel assembly at $0.15 \mathrm{GWd} / \mathrm{tHM}$.

\begin{tabular}{|c|c|c|c|c|}
\hline \multicolumn{5}{|c|}{ Fuel temperature $=900 \mathrm{~K} ;$ moderator temperature $=580 \mathrm{~K} ;$ boron concentration $=1000 \mathrm{ppm}$} \\
\hline & Group & Transport & Absorption & $\mathrm{Nu} *$ Fission \\
\hline Reference & 1 & $2.35770 \mathrm{E}-01$ & $1.19080 \mathrm{E}-02$ & 8.69247E-03 \\
\hline SCALE/TRITON & 1 & $2.13992 \mathrm{E}-01$ & $1.22269 \mathrm{E}-02$ & 8.79182E-03 \\
\hline SERPENT & 1 & $2.12058 \mathrm{E}-01$ & $1.20318 \mathrm{E}-02$ & 8.66444E-03 \\
\hline Reference & 2 & $8.67568 \mathrm{E}-01$ & $2.59391 \mathrm{E}-01$ & $3.84971 \mathrm{E}-01$ \\
\hline SCALE/TRITON & 2 & $1.00691 \mathrm{E}+00$ & $2.47747 \mathrm{E}-01$ & $3.75525 \mathrm{E}-01$ \\
\hline SERPENT & 2 & $1.02981 \mathrm{E}+00$ & 2.58149E-01 & 3.81837E-01 \\
\hline \multicolumn{5}{|c|}{ Fuel temperature $=900 \mathrm{~K} ;$ moderator temperature $=580 \mathrm{~K} ;$ boron concentration $=0 \mathrm{ppm}$} \\
\hline Reference & 1 & $2.36351 \mathrm{E}-01$ & $1.16812 \mathrm{E}-02$ & 8.68147E-03 \\
\hline SCALE/TRITON & 1 & $2.14070 \mathrm{E}-01$ & $1.20587 \mathrm{E}-02$ & 8.80908E-03 \\
\hline SERPENT & 1 & $2.11620 \mathrm{E}-01$ & $1.17854 \mathrm{E}-02$ & 8.64882E-03 \\
\hline Reference & 2 & $8.70020 \mathrm{E}-01$ & $2.49788 \mathrm{E}-01$ & 3.84959E-01 \\
\hline SCALE/TRITON & 2 & $1.00543 \mathrm{E}+00$ & $2.37959 \mathrm{E}-01$ & $3.75572 \mathrm{E}-01$ \\
\hline SERPENT & 2 & $1.02766 \mathrm{E}+00$ & $2.48512 \mathrm{E}-01$ & 3.81796E-01 \\
\hline \multicolumn{5}{|c|}{ Fuel temperature $=900 \mathrm{~K} ;$ moderator temperature $=560 \mathrm{~K} ;$ boron concentration $=1000 \mathrm{ppm}$} \\
\hline Reference & 1 & $2.41967 \mathrm{E}-01$ & $1.20423 \mathrm{E}-02$ & $8.75620 \mathrm{E}-03$ \\
\hline SCALE/TRITON & 1 & $2.18814 \mathrm{E}-01$ & $1.23548 \mathrm{E}-02$ & 8.85498E-03 \\
\hline SERPENT & 1 & $2.16841 \mathrm{E}-01$ & $1.21515 \mathrm{E}-02$ & 8.72998E-03 \\
\hline Reference & 2 & $9.09042 \mathrm{E}-01$ & $2.60681 \mathrm{E}-01$ & $3.85286 \mathrm{E}-01$ \\
\hline SCALE/TRITON & 2 & $1.05220 \mathrm{E}+00$ & $2.48887 \mathrm{E}-01$ & 3.75874E-01 \\
\hline SERPENT & 2 & $1.07675 \mathrm{E}+00$ & $2.59374 \mathrm{E}-01$ & 3.82098E-01 \\
\hline \multicolumn{5}{|c|}{ Fuel temperature $=560 \mathrm{~K} ;$ moderator temperature $=580 \mathrm{~K} ;$ boron concentration $=1000 \mathrm{ppm}$} \\
\hline Reference & 1 & $2.35975 \mathrm{E}-01$ & $1.16577 \mathrm{E}-02$ & 8.66383E-03 \\
\hline SCALE/TRITON & 1 & $2.14109 \mathrm{E}-01$ & $1.19927 \mathrm{E}-02$ & 8.77189E-03 \\
\hline SERPENT & 1 & $2.12003 \mathrm{E}-01$ & $1.17839 \mathrm{E}-02$ & $8.63921 \mathrm{E}-03$ \\
\hline Reference & 2 & $8.66972 \mathrm{E}-01$ & $2.58515 \mathrm{E}-01$ & $3.83650 \mathrm{E}-01$ \\
\hline SCALE/TRITON & 2 & $1.00585 \mathrm{E}+00$ & $2.46822 \mathrm{E}-01$ & 3.74099E-01 \\
\hline SERPENT & 2 & $1.0286 \mathrm{E}+00$ & $2.57242 \mathrm{E}-01$ & $3.80511 \mathrm{E}-01$ \\
\hline
\end{tabular}

Table 5. Two-group cross sections of the PWR MOX 4.3\% fuel assembly at $37.5 \mathrm{GWd} / \mathrm{tHM}$.

\begin{tabular}{|l|c|c|c|c|}
\hline \multicolumn{4}{|c|}{ Fuel temperature $\mathbf{9 0 0 K}$; moderator temperature $=\mathbf{5 8 0 K}$; boron concentration $=\mathbf{1 0 0 0}$ ppm } \\
\hline & Group & Transport & Absorption & Nu*Fission \\
\hline Reference & 1 & $2.33007 \mathrm{E}-01$ & $1.28084 \mathrm{E}-02$ & $6.61114 \mathrm{E}-03$ \\
\hline SCALE/TRITON & 1 & $2.16238 \mathrm{E}-01$ & $1.28002 \mathrm{E}-02$ & $6.58074 \mathrm{E}-03$ \\
\hline SERPENT & 1 & $2.16019 \mathrm{E}-01$ & $1.29332 \mathrm{E}-02$ & $6.56501 \mathrm{E}-03$ \\
\hline Reference & 2 & $8.61280 \mathrm{E}-01$ & $1.74961 \mathrm{E}-01$ & $2.44135 \mathrm{E}-01$ \\
\hline SCALE/TRITON & 2 & $9.52962 \mathrm{E}-01$ & $1.66973 \mathrm{E}-01$ & $2.42330 \mathrm{E}-01$ \\
\hline SERPENT & 2 & $9.75833 \mathrm{E}-01$ & $1.75013 \mathrm{E}-01$ & $2.43025 \mathrm{E}-01$ \\
\hline \multicolumn{2}{|c|}{ Fuel temperature $\mathbf{9 0 0 K}$; moderator temperature $=\mathbf{5 8 0 K}$; boron concentration = 0 ppm } \\
\hline Reference & 1 & $2.33935 \mathrm{E}-01$ & $1.25608 \mathrm{E}-02$ & $6.59757 \mathrm{E}-03$ \\
\hline SCALE/TRITON & 1 & $2.16316 \mathrm{E}-01$ & $1.26446 \mathrm{E}-02$ & $6.59127 \mathrm{E}-03$ \\
\hline SERPENT & 1 & $2.15516 \mathrm{E}-01$ & $1.26782 \mathrm{E}-02$ & $6.54623 \mathrm{E}-03$ \\
\hline Reference & 2 & $8.65373 \mathrm{E}-01$ & $1.65306 \mathrm{E}-01$ & $2.44058 \mathrm{E}-01$ \\
\hline SCALE/TRITON & 2 & $9.52693 \mathrm{E}-01$ & $1.57022 \mathrm{E}-01$ & $2.42193 \mathrm{E}-01$ \\
\hline SERPENT & 2 & $9.75500 \mathrm{E}-01$ & $1.65293 \mathrm{E}-01$ & $2.42855 \mathrm{E}-01$ \\
\hline \multicolumn{2}{|c|}{ Fuel temperature } \\
\hline
\end{tabular}




\begin{tabular}{|l|c|c|c|c|}
\hline Reference & 1 & $2.39039 \mathrm{E}-01$ & $1.29656 \mathrm{E}-02$ & $6.65932 \mathrm{E}-03$ \\
\hline SCALE/TRITON & 1 & $2.21069 \mathrm{E}-01$ & $1.29521 \mathrm{E}-02$ & $6.63079 \mathrm{E}-03$ \\
\hline SERPENT & 1 & $2.20795 \mathrm{E}-01$ & $1.30968 \mathrm{E}-02$ & $6.61205 \mathrm{E}-03$ \\
\hline Reference & 2 & $9.04032 \mathrm{E}-01$ & $1.76043 \mathrm{E}-01$ & $2.43855 \mathrm{E}-01$ \\
\hline SCALE/TRITON & 2 & $9.98085 \mathrm{E}-01$ & $1.67841 \mathrm{E}-01$ & $2.41970 \mathrm{E}-01$ \\
\hline SERPENT & 2 & $1.02354 \mathrm{E}+00$ & $1.75986 \mathrm{E}-01$ & $2.42539 \mathrm{E}-01$ \\
\hline \multicolumn{2}{|c|}{ Fuel temperature } & $\mathbf{5 6 0 K}$; moderator temperature $=\mathbf{5 8 0 K}$; boron concentration $=\mathbf{1 0 0 0} \mathbf{p p m}$ \\
\hline Reference & 1 & $2.33242 \mathrm{E}-01$ & $1.25739 \mathrm{E}-02$ & $6.61576 \mathrm{E}-03$ \\
\hline SCALE/TRITON & 1 & $2.16346 \mathrm{e}-01$ & $1.25904 \mathrm{E}-02$ & $6.59333 \mathrm{E}-03$ \\
\hline SERPENT & 1 & $2.15998 \mathrm{E}-01$ & $1.27139 \mathrm{E}-02$ & $6.56759 \mathrm{E}-03$ \\
\hline Reference & 2 & $8.61038 \mathrm{E}-01$ & $1.74513 \mathrm{E}-01$ & $2.43256 \mathrm{E}-01$ \\
\hline SCALE/TRITON & 2 & $9.52453 \mathrm{E}-01$ & $1.66405 \mathrm{E}-01$ & $2.41354 \mathrm{E}-01$ \\
\hline SERPENT & 2 & $9.76395 \mathrm{E}-01$ & $1.74575 \mathrm{E}-01$ & $2.42145 \mathrm{E}-01$ \\
\hline
\end{tabular}

\section{Conclusions}

In this study, the SCALE/TRITON (deterministic method) and Serpent (Monte Carlo method) codes were utilized to generate the homogenized two-group neutron cross sections for the PWR MOX $4.0 \%$ and $4.3 \%$ fuel assemblies. The purpose was to use both the deterministic and Monte Carlo methods to prepare the group constants for analyzing REAs in PWR MOX fueled cores using 3D reactor kinetics codes like PARCS and NODAL3. The k-inf values of the PWR MOX assemblies versus burnup and the respective two-group neutron cross sections obtained with TRITON and Serpent were analyzed and compared with the reference benchmark data obtained with the HELIOS code. The comparative results generally exhibit a good agreement between TRITON and Serpent with HELIOS within $643 \mathrm{pcm}$ for the k-inf values and within 5\% for the two-group neutron cross sections, demonstrating the reliability of the TRITON and Serpent models developed in the present work for generating the homogenized fewgroup constants of the PWR MOX assemblies. The discrepancy between TRITON, Serpent and HELIOS, especially in predicting the two-group transport cross sections of the PWR MOX assemblies, might be mainly because of different calculation methods and nuclear data libraries employed in each code. It is also worth noting that the deterministic and Monte Carlo models with TRITON and Serpent for the PWR MOX assemblies developed have the advantages of branch calculations which could facilitate the group constant generation for any change in the operating conditions of the PWR MOX fueled cores, such as fuel burnup, moving of control rods, and changes in the fuel temperature, moderator density and temperature and boron concentration. These models with TRITON and Serpent are being further improved and applied to serve for control rod ejection transient analyses of PWR MOX fueled cores using 3D reactor kinetics codes.

\section{Acknowledgments}

This research is funded by Vietnam National Foundation for Science and Technology Development (NAFOSTED) under Grant 103.99-2018.32.

\section{References}

[1] T. Kozlowski, T. J. Downar, PWR MOX/UO 2 Core Transient Benchmark Final Report, Purdue University, ISBN 92-64-02330-5, NEA/NSC/DOC 20, US, 2006. 
[2] T. J. Downar, Y. Xu, V. Seker, N. Hudson, User Manual for the PARCS v3.0 U.S. NRC Core Neutronics Simulator, University of Michigan, UM-NERS-09-0001, US, 2012.

[3] S. Pinem, T. M. Sembiring, P. H. Liem, The Verification of Coupled Neutronics Thermal-Hydraulics Code NODAL3 in the PWR Rod Ejection Benchmark, Science and Technology of Nuclear Installations, Vol. 2014, 2014, Article ID 845832, https://doi.org/10.1155/2014/845832.

[4] B. T. Rearden , M. A. Jessee (Editors), SCALE Code System User Manual (Version 6.2.2), Oak Ridge National Laboratory, ORNL/TM-2005/39, US, 2017.

[5] J. Leppänen, M. Pusa, T. Viitanen, V. Valtavirta, T. Kaltiaisenaho, The Serpent Monte Carlo Code: Status, Development and Applications in 2013, Annals of Nuclear Energy, Vol. 82, 2015, pp. 142-150, https://doi.org/10.1016/j.anucene.2014.08.024.

[6] J. Leppänen, M. Pusa, E. Fridman, Overview of Methodology for Spatial Homogenization in the Serpent 2 Monte Carlo Code, Annals of Nuclear Energy, Vol. 96, 2016, pp. 126-136, https://doi.org/10.1016/j.anucene.2016.06.007.

[7] Z. Liu, K. Smith, B. Forget, J. Ortensi, Cumulative Migration Method for Computing Rigorous Diffusion Coefficients and Transport Cross Sections from Monte Carlo, Annals of Nuclear Energy, Vol. 112, 2018, pp. 507516, https://doi.org/10.1016/j.anucene.2017.10.039.

[8] J. Leppänen, Input Syntax Manual, http://serpent.vtt.fi/mediawiki/index.php/Input_syntax_manual, 2020 (accessed on: August $\left.6^{\text {th }}, 2020\right)$.

[9] M. B. Chadwick, P. Obložinský, M. Herman, N. M. Greene, R. D. McKnight, D. L. Smith, P. G. Young, R. E. MacFarlane, G. M. Hale, S. C. Frankle, A. C. Kahler, T. Kawano, R. C. Little, D. G. Madland, P. Moller, R. D. Mosteller, P. R. Page, P. Talou, H. Trellue, M. C. White, W. B. Wilson, R. Arcilla, C. L. Dunford, S. F. Mughabghab, B. Pritychenko, D. Rochman, A. A. Sonzogni, C. R. Lubitz, T. H. Trumbull, J. P. Weinman, D. A. Brown, D. E. Cullen, D. P. Heinrichs, D. P. McNabb, H. Derrien, M. E. Dunn, N. M. Larson, L. C. Leal, A. D. Carlson, R. C. Block, J. B. Briggs, E. T. Cheng, H. C. Huria, M. L. Zerkle, K. S. Kozier, A. Courcelle, V. Pronyaev, S. C. van der Marck, ENDF/B-VII.0: Next Generation Evaluated Nuclear Data Library for Nuclear Science and Technology, Nuclear Data Sheets, Vol. 107, No. 12, 2006, pp. 2931-3060, https://doi.org/10.1016/j.nds.2006.11.001.

[10] J. Leppänen, WIMS 172-group Structure, http://serpent.vtt.fi/mediawiki/index.php/WIMS_172-group_structure, 2020 (accessed on: August $6^{\text {th }}, 2020$ ). 